\title{
La face cachée de Newton : antitrinitarien et alchimiste
}

The Hidden Face of Newton: Antitrinitarian and Alchemist

Jean Rivière

\section{(2) OpenEdition}

1 Journals

\section{Édition électronique}

URL : http://journals.openedition.org/rfcb/3583

DOI : $10.4000 / \mathrm{rfcb} .3583$

ISSN : 2429-4373

Éditeur

CRECIB - Centre de recherche et d'études en civilisation britannique

\section{Édition imprimée}

Date de publication : 1 mars 2013

ISBN : 2-911580-37-0

ISSN : 0248-9015

\section{Référence électronique}

Jean Rivière, "La face cachée de Newton : antitrinitarien et alchimiste ", Revue Française de Civilisation Britannique [En ligne], XVIII-1 | 2013, mis en ligne le 01 mars 2013, consulté le 20 mars 2020. URL http://journals.openedition.org/rfcb/3583 ; DOI : https://doi.org/10.4000/rfcb.3583

Ce document a été généré automatiquement le 20 mars 2020.

\section{cc) (†)}

Revue française de civilisation britannique est mis à disposition selon les termes de la licence Creative Commons Attribution - Pas d'Utilisation Commerciale - Pas de Modification 4.0 International. 


\title{
La face cachée de Newton : antitrinitarien et alchimiste
}

The Hidden Face of Newton: Antitrinitarian and Alchemist

\author{
Jean Rivière
}

1 Dans l'Europe tourmentée de la Réforme protestante et de la Contre-Réforme catholique, les allégeances confessionnelles prennent le pas sur toutes les autres. La paix religieuse qui s'établit après 1648 est fondée sur le principe suivant: les sujets adoptent la religion de leur souverain, et s'ils ne peuvent s'y résoudre, ils se font plus ou moins discrets. Telle est la solution qui prévaut en Allemagne. Dans les autres pays, les lignes sont beaucoup plus tranchées. En Espagne, en Italie et au Portugal, la Réforme s'effondre sous les coups de l'Inquisition. Au Nord, les réformes luthériennes se font sous l'égide des familles princières. En France, c'est le retour au statu quo ante avec la Révocation de l'Édit de Nantes en 1685.

Dans l'Angleterre des années 1660, le système épiscopal supprimé sous Cromwell est rétabli sous Charles II, l'Église d'Angleterre redevenant l'institution religieuse officielle, dont la doctrine, à mi-chemin entre le calvinisme et le catholicisme, est exposée dans les Trente-Neuf Articles. Ceux qui ne s'y conforment pas acquièrent le statut officieux de non-conformiste. S'ils veulent bénéficier des mêmes droits que les anglicans pour accéder aux emplois publics et aux universités, ils doivent se conformer - au moins occasionnellement - au rite anglican en communiant dans une église de cette obédience. Cette démarche, reconnue ou tolérée, est sujette à variation.

La principale stratégie pour les non-conformistes, les libertins et ceux qu'on appelle en France les "esprits forts", est de dissimuler: cette attitude prévaut dans les pays protestants pour les catholiques, dans les pays catholiques pour les protestants et, pendant tout le XVI ${ }^{\mathrm{e}}$ siècle, pour les juifs dans la péninsule ibérique ; elle prend le nom de "nicodémisme " - d'après le disciple Nicodème, qui, dans l'évangile de Jean, se conformait aux rites juifs le jour et participait aux réunions chrétiennes la nuit. On ne saurait oublier que le dialogue est impossible entre les tenants de l'orthodoxie locale et ceux qui la contestent: ainsi, lorsque Pascal, dans son célèbre pari, s'adresse à un incroyant, ce dernier ne peut lui répondre sous peine de mettre en jeu sa liberté, voire 
sa vie. L'Inquisition romaine est rétablie en 1542, et ses méthodes sont tout naturellement adoptées dans le camp de la Réforme. Ainsi, Michel Servet, ce médecin espagnol qui a échappé à l'Inquisition dans son pays, est brûlé vif à Genève en 1553 sur l'ordre de Calvin pour avoir nié la Trinité et le péché originel. Seules les ProvincesUnies sont un refuge sûr pour les juifs (encore ne faut-il pas qu'ils passent les bornes dans leurs écrits, comme Spinoza, excommunié par sa propre communauté religieuse), les protestants et les jansénistes. Passer d'un pays à l'autre peut contribuer à se faire oublier dans un lieu où votre "réputation" est inconnue. Ainsi, Thomas Hobbes (1588-1679), philosophe mécaniste et matérialiste, et partisan d'un pouvoir politique absolu dans une société où l'homme est un loup pour l'homme, séjourne en France de 1640 à 1651 pour échapper au pouvoir puritain mis en place en Angleterre. Il y fréquente le cercle du père Marin Mersenne et entre en contact ou en correspondance épistolaire avec des penseurs tels que Fermat, Pascal, Descartes ou encore Torricelli. Grâce à Mersenne, il peut lire une première version des Méditations métaphysiques de Descartes. À l'inverse, le Français Charles de Saint-Evremond (1614-1703), « esprit fort » et libertin, passe les quarante-deux dernières années de sa vie en Angleterre, mis à part un séjour de 1665 à 1670 dans les Provinces-Unies, où il rencontre Spinoza. Ses écrits, franchement déistes, et défendant une séparation totale entre religion et pouvoir politique, sont publiés - la plupart du temps clandestinement - à Amsterdam ou en France. Il est le seul Français à être enterré à Westminster Abbey et à avoir sa plaque dans le Poets' Corner. Ces deux esprits illustrent à merveille le proverbe selon lequel « nul n'est prophète en son pays ». En politique, Machiavel est par excellence le tenant de la dissimulation, stratégie obligatoire pour le prince qui veut gouverner et atteindre le but qu'il s'est fixé. En littérature, le théâtre de Shakespeare n'est qu'une suite de dissimulations d'identité et de desseins secrets pour mieux arriver à ses fins. Ainsi, Hamlet dissimule pendant la plus grande partie de la pièce ce qu'il sait (que c'est son oncle Claudius qui a assassiné son père) et son intention de tuer Claudius au moment qu'il jugera opportun. Il feindra même la folie pour mieux mettre son plan à exécution. Montaigne est très clair dans ses Essais: « Aux Français le mentir et se parjurer n'est pas vice [...] ce leur est à présent vertu. On s'y forme, on s'y façonne, comme à un exercice d'honneur ; car la dissimulation est des plus notables qualitez de ce siècle $»^{1} . .$. et du suivant ${ }^{2}$.

\section{Newton et l'antitrinitarisme}

4 Newton entra comme étudiant à Cambridge , à Trinity College plus précisément, en 1661, et y resta jusqu'en 1701, passant donc la plus grande partie d'une longue carrière dans cette université de l'élite aristocratique et intellectuelle, où il fut tour à tour étudiant, diplômé, fellow et professeur de plein exercice. Les différents grades qu'il obtint à Cambridge l'obligèrent, chaque fois, à prêter un serment d'allégeance à la doctrine de l'Église anglicane (graduate, fellow) selon l'Act of Conformity (1662). Pour certains emplois universitaires, comme les chaires de professeur, il fallait en outre recevoir les ordres anglicans.

Depuis 1672, Newton avait distrait une bonne partie de son temps de chercheur pour s'occuper de théologie, en particulier du dogme de la Trinité. Celui-ci avait été contesté dès les premiers siècles de l'Église par les ariens, qui ne reconnaissaient pas la divinité du Christ, et avaient failli l'emporter au Concile de Nicée. Arius (256-336 après J.- C.) croyait que, dans la Trinité, le Fils n'était pas parfaitement égal au Père. Le Christ était 
bien le Fils de Dieu conçu de la Vierge Marie, mais n'était pas Dieu lui-même. C'est la controverse introduite dans toute l'Église par la doctrine et la prédication d'Arius qui poussa l'empereur Constantin à convoquer en 325 le Concile de Nicée, qui affirma la parfaite égalité divine entre le Père, le Fils et le Saint-Esprit. La polémique n'en fut pas apaisée, et c'est seulement en 381, au Concile de Constantinople, que la doctrine de Nicée fut officiellement reconnue. Pourtant, l'arianisme survécut encore longtemps chez les Barbares (Goths, Vandales, Burgondes) en Italie, en Gaule, en Espagne et en Afrique, et ce n'est qu'avec la conversion des Francs à la fin du V $\mathrm{V}^{\mathrm{e}}$ siècle, et celle des Lombards au VII ${ }^{e}$ siècle à l'orthodoxie romaine que l'arianisme disparut au sein de l'Église.

6 Pour Newton, l'étude objective des quatre évangiles canoniques semblait corroborer cette interprétation arienne: Jésus y est constamment désigné comme le fils de l'Homme, ou le fils de Dieu, jamais comme Dieu lui-même. À une époque comme la nôtre, où la pratique religieuse est confrontée à une offre pléthorique, ces distinctions peuvent sembler dérisoires. Et pourtant, en plein XIX ${ }^{e}$ siècle, en France, Renan fut révoqué de sa chaire au Collège de France en 1864 parce que, dans sa leçon inaugurale, il avait parlé de Jésus, « cet homme admirable ». Certes, à cette époque, la majorité de la classe intellectuelle française ne croyait plus en la Trinité, mais le gouvernement impérial d'alors ne pouvait se permettre de laisser bafouer ouvertement ce dogme dans un pays encore officiellement catholique.

7 Newton était convaincu que les deux passages où il avait trouvé une référence au Dieu en trois personnes, toutes trois pleinement divines, c'est-à-dire 1 Jean $5: 7^{3}$ et 1Timothée 3:164, étaient des interpolations de l'Église romaine. En 1672, il inscrit dans un carnet les problèmes religieux qu'il compte étudier : attributs de Dieu, Dieu le Fils, l'Incarnation. Il recense tous les passages du Nouveau Testament où le Fils est subordonné au Père. Il a donc bien déjà des doutes sur la divinité du Christ. D'autre part, dans son étude des Pères de l'Église, il note les très nombreux passages infirmant la divinité du Fils, sans toutefois publier les conclusions antitrinitariennes qu'il en tire. Pour Newton, le culte du Christ comme Dieu est de l'idolâtrie, et l'Église catholique, son suppôt principal, une secte idolâtre. Newton griffonna ou rédigea des centaines de pages sur le sujet. Pour lui, la Réforme n'était pas allée au fond du problème, puisqu'elle avait adhéré à une doctrine trinitaire pleine et entière. Il aurait fallu, selon lui, considérer le Père comme le Dieu suprême et unique, et le Christ comme un logos (dans la tradition de l'apôtre Jean) par le truchement duquel parlait Dieu le Père. Entre 1672 et 1675 , Newton résume ainsi sa doctrine sur le sujet : le mot « Dieu » employé seul dans les Écritures se réfère à Dieu le Père, et la référence à un seul Dieu l'est toujours à Dieu le Père ; le Christ appelle Dieu le Père son Dieu, et la prescience de toute chose à venir appartient uniquement au Père; les pouvoirs que peut détenir le Fils ne peuvent donc être dérivés que de ceux que possède le Père. Cette doctrine, même si elle ne fut jamais exposée officiellement, allait compliquer les relations de Newton avec le Cambridge de son époque.

8 Cambridge était certes une institution tolérante dans le domaine de la recherche scientifique, mais elle n'aurait admis aucune expression orale ou écrite d'une proposition hétérodoxe. Ainsi Daniel Scargill en fut-il exclu en 1669 « pour avoir soutenu des dogmes impies et athées » et n'y fut-il réintégré qu'après une abjuration publique à la manière de Galilée. Isaac Barrow, le prédécesseur de Newton à la chaire de mathématique, avait jugé prudent de défendre le dogme de la Trinité dans l'un de ses 
sermons ${ }^{5}$. Et puis, en 1675 , se posait une question cruciale pour Newton: si l'on ne changeait pas les règles d'accession à la chaire de mathématique, il devrait recevoir les ordres anglicans. Trois enseignants de Trinity College avaient dû démissionner entre 1661 et 1666 pour avoir refusé de se soumettre à cette règle. Newton évita l'obstacle de l'ordination grâce à l'entremise d'Isaac Barrow. Il fallait pour cela qu'il eût l'oreille des plus hautes autorités gouvernementales. En 1675, il obtint finalement de Charles II l'autorisation de ne pas être soumis à l'obligation d'ordination afin d'occuper la chaire de mathématique, «à moins qu'il ne le désire ». Suivant les termes de la décision royale, la dispense avait pour but d' "accorder tout encouragement juste aux savants destinés à être élus à la dite chaire ". La dispense fut accordée à perpétuité. Newton avait donc su naviguer au plus près pour accéder à son poste, sans lequel il aurait été privé de tout moyen financier autonome pour poursuivre ses recherches. Certes, le contrôle ecclésiastique sur la recherche en Angleterre n'avait rien à voir avec les foudres de l'Inquisition romaine, mais le cas prouvait qu'il existait encore dans le monde universitaire anglais de la fin du XVII ${ }^{e}$ siècle les moyens de faire taire, au moins en les privant de ressources, les esprits rétifs à une orthodoxie sans faille. Il valait mieux cacher aux autres, voire à soi-même, ses propres écarts par rapport à la doctrine officielle.

9 Rien ne transpira donc de son vivant des déviations théologiques de Newton, qui ne furent connues qu'en 1754 lors de la publication posthume de An Historical Account of Two Notable Corruptions of Scripture ! S'il avait fait connaître ses opinions hétérodoxes, il aurait perdu son poste ; s'il les avait publiées, il aurait pu connaître la paille humide des cachots pour une durée indéterminée. Ainsi, le plus grand savant de l'ère moderne, au sens historique du terme, ne pouvait pas exposer une déviation - somme toute vénielle en apparence - par rapport à l'orthodoxie traditionnelle, et ce, dans le pays le plus libéral de l'époque avec la Suède et les Provinces-Unies. Faute vénielle en apparence seulement, car si la Trinité n'était plus reconnue comme un dogme fondateur du christianisme, l'Incarnation et la Rédemption disparaissaient par la même occasion.

Newton poursuivit ses études antitrinitariennes tout au long de sa vie et, en particulier, dans sa correspondance avec John Locke, en ayant toutefois la prudence d'adresser ses missives à un "ami», et non à Locke en personne. Pour Newton, il est urgent d'expurger les textes bibliques afin qu'ils reflètent enfin une vérité qui, à ses yeux, ne souffre pas la controverse, mais, prudent, il enjoint à Locke de ne pas divulguer ses remarques " hérétiques » sur les vingt-sept passages qu'il soupçonne d'avoir été ajoutés au texte originel de la Bible et aux écrits des Pères de l'Église. Il lui déclare surtout que le grand public ignorant est friand de mystères. Ainsi, le plus prestigieux savant de son époque, Isaac Newton, et l'un des plus grands philosophes de son temps, John Locke, durent déployer toutes sortes de ruses pour échapper à leurs censeurs ${ }^{6}$.

\section{L'alchimie ou le rêve secret de Newton}

11 L'alchimie peut être considérée non comme une pseudoscience, mais comme une protoscience. Pendant plusieurs millénaires, en effet, elle a rempli une sorte de vide, alors que l'on ignorait la structure et les lois de transformation de la matière. Certes, Démocrite, 500 ans avant notre ère, avait énoncé une théorie atomique, mais ses particules se situaient en dehors du champ d'investigation des savants de l'époque. De même que l'astrologie - prédiction de l'avenir d'après le mouvement des astres - avait 
précédé l'astronomie - étude objective de l'univers -, la magie, de son côté, avait précédé la médecine d'Hippocrate et de Gallien. On trouve des traces de recherches alchimiques dans toutes les civilisations orientales et extrême-orientales et, naturellement, en Occident jusqu'aux découvertes pionnières du XVIII ${ }^{\mathrm{e}}$ siècle sur la nature de la chimie. L'alchimie reposait sur deux rêves : le premier est l'élixir de longue vie, qui permettrait à l'homme de vivre plus longtemps, voire éternellement, et le second est la pierre philosophale, qui nous rendrait capables de transformer tous les métaux vils en or.

Dans ses Questiones, Newton avait noté dès 1666 les différentes opérations qui recouvraient le domaine de la chimie : l'« abstraction », par exemple, consistait à faire évaporer et à distiller une solution pour en extraire le sel. Les termes "creuset", « extraction» et «amalgame» figurent également dans ses cahiers. Sous la rubrique " expérimenter ", il décrit la façon de raffiner l'or et l'argent en les chauffant avec du plomb. Newton fit partie de la très clandestine Société des Alchimistes Anglais et, par l'intermédiaire de cette dernière, put se procurer de nombreux ouvrages, dont le Theatrum Magicum. Il annota ces écrits pendant une trentaine d'années. Il ne s'agit donc nullement pour lui d'une lubie passagère, mais d'une démarche continue pour mieux cerner la nature de la matière et l'utiliser dans un but expérimental. On peut considérer que Newton fut le plus grand alchimiste de son temps, car il apporta à ses recherches, hors du champ scientifique proprement dit, la détermination dont il savait faire preuve comme physicien, astronome et mathématicien.

13 Quelle pouvait être la signification de l'alchimie pour Newton? À une époque où la chimie n'était que balbutiante, l'alchimie avait pour avantage d'offrir quelques perspectives de synthèse sur la nature de la matière et la façon de la transformer. L'expérimentation chimique pouvait donc coexister avec la spéculation alchimique. Le mercure avait ici une importance primordiale, car on le considérait comme l'élément constitutif des autres métaux : il fallait le débarrasser de ses scories impures pour le rendre propre aux opérations visant à sa transformation en d'autres métaux. Comme le but de l'alchimie était la fabrication d'or, on aurait pu croire que Newton se serait dirigé vers des tentatives expérimentales dans ce domaine. Mais le savant qu'il était avait trouvé dans l'alchimie une nouvelle approche de la démarche scientifique. Alors que les mécanistes séparaient le corps de l'esprit, montrant que l'univers se mouvait suivant des lois indépendantes de l'homme, les alchimistes considéraient que la matière était dotée d'une vie propre. Ainsi la pierre était-elle composée d'un corps (imparfait), d'une âme (donnant vie à ce corps imparfait) et d'un esprit (l'eau). Alors que la philosophie mécaniste insistait sur l'inertie de la matière, que seuls le mouvement et la gravitation pouvaient mettre en branle, l'alchimie reposait sur un principe actif : la pierre philosophale traditionnelle, dont le magnétisme constituait la qualité essentielle.

Ainsi Newton entra-t-il dans un corps secret : celui des alchimistes anglais. Il se forgea à cette occasion un pseudonyme, Jeova sanctus unus, anagramme d'Isaacus Neuutonus, en latin, et conduisit un nombre incalculable d'expériences alchimiques à Trinity College, dans un laboratoire de fortune : un appentis qui se trouvait dans son jardin et jouxtait la chapelle. Cette proximité n'était pas fortuite, car, pour les alchimistes, la transmutation des métaux signifiait une purification spirituelle. C'était Dieu qui insufflait la vie à la matière, comme aux premiers temps de la Création. Il n'est donc pas étonnant que les recherches alchimiques de Newton se soient poursuivies dans les années où il se plongeait le plus intensément dans l'étude de la Bible et des Pères de 
l'Église. Plutôt que de se détourner de ce qu'il ne pouvait pas comprendre, il se plongeait dans les observations et les expériences les plus hétéroclites pour se mettre en rapport avec les forces végétales, vitales et sexuelles qui peuplaient notre univers. Fourneaux et creusets devenaient ses outils privilégiés pour atteindre des secrets de l'univers sur lesquels sa science mécaniste ne pouvait l'éclairer. À la fin du XVII siècle, il s'était ainsi construit un Index chemicus, manuscrit de plus de cent pages, comprenant plus de cinq mille références à des ouvrages alchimiques des époques antérieures. Ses propres écrits ne furent publiés que des années après sa mort. Si l'on additionne tous ses ouvrages imprimés et ses manuscrits sur l'alchimie, on arrive à plus d'un million de mots!

15 Avec sa théorie de la gravitation universelle et de la mécanique qui en découlait, Newton, en étudiant la matière, pensait que les principes mécaniques ne pouvaient dans ce cas s'appliquer tels quels. La mécanique ne suffisait pas à rendre compte des mouvements de la matière. Certes, Descartes avait bien énoncé une "théorie des tourbillons » qui faisait se mouvoir la matière, mais Newton s'inscrivit très tôt en faux contre cette thèse. L'alchimie lui offrait des perspectives élargies de compréhension et de transformation de la matière, mais il allait falloir attendre encore près d'un siècle pour que la chimie se dotât de ses propres critères objectifs et scientifiques, l'essentiel étant que, dans la nature, rien ne se perd, et rien ne se crée. Pour Newton, l'alchimie ne représente pas une démarche contraire aux lois de la mécanique, mais une pratique qui nous permet de mieux comprendre la matière pour la transformer, sans verser dans le rêve fou des alchimistes de pouvoir convertir toute matière en or. Newton occupe ainsi une position intermédiaire entre des lois mécaniques trop vastes et trop générales pour étudier ce champ particulier et des lois chimiques encore à découvrir. On peut surtout penser que ses études alchimiques constituaient une révolte de son esprit contre les limites que la pensée des mécanistes comme Descartes introduisait dans la philosophie naturelle. Trente années de passions insatisfaites de 1670 à 1700 - puisque ses expériences alchimiques ne le menèrent jamais à aucune découverte d'envergure - ne l'empêchèrent pas de considérer que, d'une certaine manière, le jeu en valait bien la chandelle?

\section{Vers une philosophie naturelle et une science chimique}

Les recherches théologiques de Newton peuvent sembler aujourd'hui ne porter que sur des détails, mais nous avons vu comment le rejet du dogme de la Trinité allait conduire au XVIII ${ }^{e}$ siècle à une remise en cause progressive de toute la dogmatique chrétienne, tandis que la croyance dans les miracles semblait une insulte à la pensée rationnelle. La répudiation des prophéties allait constituer la troisième étape vers une religion déiste, telle que l'exposait Thomas Paine à la fin du Siècle des Lumières dans The Age of Reason (1795). Philosophie naturelle et religion naturelle en viennent donc à répudier dogmes, miracles et prophéties en bloc, ce que ne fait pas encore Newton.

Pour ce qui est de la chimie, cette science encore balbutiante avait eu deux précurseurs, que Newton n'a pas suivis: le premier est le Flamand Jan Baptist Van Helmont (1579-1644), qui fut chimiste et médecin, mais aussi alchimiste. Il créa la notion de "gaz ", et découvrit le gaz carbonique et les acides sulfhydrique et chlorhydrique. Il reconnut le suc gastrique comme un agent actif de la digestion et créa un thermomètre 
en utilisant la dilatation de l'eau dans une enveloppe de verre. Sir Robert Boyle (1621-1691) fut plus proche de Newton, puisqu'il fut l'un des fondateurs de la Royal Society. Il énonça avec Mariotte la loi de la compressibilité des gaz. Dans Sceptical Chymist (1661), il rejette la théorie des éléments d'Aristote et fait apparaître la notion d'élément chimique. Il reconnaît le rôle de l'oxygène dans la combustion et la respiration. Mais ce n'est qu'avec Lavoisier (1743-1794) que s'établirent les bases de la chimie: composition de l'air et de l'eau, distinction entre corps simples et combinaisons, mécanismes des combustions.

On peut donc conclure que Newton a œuvré dans le champ théologique en y pointant des contradictions qui allaient être pleinement reconnues au XVIII ${ }^{e}$ siècle, et qu'il a exploré avec la détermination qu'on lui connaît les champs de la chimie avec les outils de l'alchimie, qui n'était qu'une protoscience.

\section{NOTES}

1. Michel de MONTAIGNE, Essais, Paris : Éditions Gallimard, 1950, chap. XVIII, « Du démentir », p. 752.

2. Pour toutes les questions de dissimulation, voir Perez ZAGORIN, Ways of Lying. Dissimulation, Persecution and Conformity in Early Modern Europe, Cambridge, Mass.: Harvard University Press, 1990. Cet ouvrage examine tous les problèmes liés à la dissimulation et à la fausse conformité chez les catholiques, les protestants, les juifs et les incroyants. On y trouve en somme un tableau de toutes les méthodes de survie possibles devant la persécution dans l'Europe occidentale des $\mathrm{XVI}^{\mathrm{e}}$ et XVII ${ }^{\mathrm{e}}$ siècles.

3. "Car il y en a trois dans le Ciel qui rendent témoignage, le Père, la Parole, et le Saint-Esprit; et ces troislà ne sont qu'un" (Bible David Martin, 1744).

4. "Dieu a été manifesté en chair, justifié en esprit, vu des Anges, prêché aux Gentils, cru au monde, et élevé dans la Gloire » (D. M.).

5. Prononcé en 1663, celui-ci est intitulé 'A Defence of the Blessed Trinity'. Il sera publié de manière posthume à Londres en 1697.

6. Voir David BERLINSKI, Newton's Gift: How Sir Isaac Newton Unlocked the System of the World, New York \& London: The Free Press, 2000, pp. 64-69 ; James GLEICH, Isaac Newton, London \& New York: Fourth Estate, 2003, chap. 10 'Heresy, blasphemy and idolatry'; Richard WESTFALL, Never at Rest. A Biography of Isaac Newton, Cambridge: Cambridge University Press, 1993, chap. 8 'Rebellion'.

7. GLEICH, op. cit, chap. 9 'All things are corruptible' ; WESTFALL, op. cit., chap. 8 ; Jean-Pierre LUMINET, La Perruque de Newton, Paris : J.-C. Lattès, 2010, chap. intitulé « Les noces chimiques ». 


\section{RÉSUMÉS}

Le plus grand savant de son époque, Newton, passa néanmoins la plus grande partie de sa vie à remettre en cause le dogme de la Trinité et mena des expériences dans le domaine de l'alchimie. Il le fit toujours de façon clandestine afin d'éviter de perdre sa chaire de professeur ... à Trinity College.

Newton, considered as the greatest scientist of his time, spent a good deal of his life challenging the dogma of the Trinity and, strangely enough, conducted experiments in the field of alchemy. He did so in the most clandestine way in order to avoid losing his professorship ... at Trinity College.

\section{AUTEUR}

\section{JEAN RIVIÈRE}

Université Paris-Dauphine 